\title{
Severe Proteinuria Secondary to Amyloidosis Requiring Bilateral Renal Artery Embolization
}

\author{
Chun-Tung Yeh ${ }^{a}$ Hsiuo-Shan Tseng ${ }^{b}$ Wen-Sheng Liu ${ }^{c, d}$ \\ Szu-Yuan $\mathrm{Li}^{\mathrm{c}}$ Wu Chang Yang ${ }^{\mathrm{c}}$ Yee-Yung $\mathrm{Ng}^{\mathrm{c}}$ \\ ${ }^{a}$ Department of Medicine, Ten-Chan General Hospital, Jhongli City, \\ ${ }^{b}$ Department of Radiology, and 'Division of Nephrology, Department of \\ Medicine, Taipei Veterans General Hospital, School of Medicine, National \\ Yang-Ming University, and ${ }^{\mathrm{d} D i v i s i o n}$ of Nephrology, Taipei City Hospital, \\ Zhongxing Branch, Taipei, Taiwan, ROC
}

\section{Key Words}

Amyloidosis · Chronic kidney disease - Proteinuria - Renal artery embolization

\begin{abstract}
In the case reported here, after prolonged medical therapy resistance, severe proteinuria subsided following bilateral renal artery embolization (RAE). Thereafter, respiratory distress, anasarca edema, muscle mass, and serum albumin level improved after regular hemodialysis. Although RAE is reported to be a safe and effective therapeutic procedure, it is rarely used for severe proteinuria with prolonged medical therapy resistance. The limited use of bilateral RAE for nephrological purposes may be partly related to its tendency to destroy renal function, which results in anuria and subsequent regular dialysis. However, delayed RAE could cause the patient to reach a life-threatening cachexic state and could increase the risk of morbidity and mortality due to severe proteinuria-induced hypoalbuminemia. Our case and selected previous reports reveal important information for physicians and patients while discussing prognoses and considering the pros and cons of bilateral RAE.
\end{abstract}

\section{Introduction}

Although renal embolization is reported to be a safe and effective therapeutic procedure for embolization of small branches of renal artery, it is mainly used for urological purposes, i.e. vascular malformations, angiomyolipomas, and renal tumors 
that are not amenable to surgical resection [1,2]. A search of the literature for the past 20 years reveals that only a few cases of renal amyloidosis [3-7] and severe nephrotic syndrome [8-11] have resulted in bilateral renal artery embolization (RAE) for severe proteinuria. The limited use of bilateral RAE for nephrological purposes may be partly related to its tendency to destroy renal function, which results in anuria and subsequent regular dialysis. Regular dialysis is usually stressful for patients [12], so some patients may be reluctant to receive bilateral RAE for severe proteinuria, which can induce hypoalbuminemia and increase the risk of morbidity and mortality $[13,14]$. Therefore, doctors and patients with renal amyloidosis and proteinuria face a difficult dilemma in deciding whether to use bilateral RAE or supportive treatments. This study reports a 66-year-old patient with renal amyloidosis and severe proteinuria, who received delayed bilateral RAE, until a life-threatening pulmonary edema occurred. Finally, bilateral RAE, followed by regular hemodialysis (HD), successfully cured the severe proteinuria and its related symptoms.

\section{Case Report}

A 66-year-old man diagnosed with chronic lymphocytic leukemia had been treated with prednisolone $5 \mathrm{mg}$ per day and cyclophosphamide $25 \mathrm{mg}$ per day since 1995. Renal amyloidosis was diagnosed after a biopsy in 2006. He was referred to our clinic because he had been suffering from renal amyloidosis-related heavy proteinuria $(21.77 \mathrm{~g} /$ day), hypoalbuminemia $(1.4 \mathrm{~g} / \mathrm{dl})$, and anasarca edema for 6 months. Because bilateral RAE was not performed, progressive anasarca edema, increased proteinuria (31.2 g/day), reduced serum albumin $(0.9 \mathrm{~g} / \mathrm{dl})$, and dyspnea developed after further conservative treatment with bed rest, salt and water restriction, diuretics such as furosemide $80 \mathrm{mg}$ and hydrochlorothiazide $50 \mathrm{mg}$ trice daily, ramipril $1.25 \mathrm{mg}$ once daily, indomethacin $25 \mathrm{mg}$ trice daily and albumin infusion, for 1 month. The serum BUN (normal range $8-20 \mathrm{mg} / \mathrm{dl}$ ) and creatinine (normal range $0.8-1.5 \mathrm{mg} / \mathrm{dl}$ ) levels were increased to 43 and $4.8 \mathrm{mg} / \mathrm{dl}$ from 36 and 3.0 $\mathrm{mg} / \mathrm{dl}$, respectively. On physical examination, body weight was $55 \mathrm{~kg}$, body height $162 \mathrm{~cm}$, blood pressure 70 80/50 60 $\mathrm{mm} \mathrm{Hg}$, pulse rate 84 beats per minute, respiratory rate 20 breaths per minute, and body temperature $37^{\circ} \mathrm{C}$. The bilateral pleural effusion and pulmonary edema gave rise to breathing difficulty. Laboratory investigation revealed white blood cells (WBC), $4.3 \times 10^{9} / 1$ (normal range 4.5-11 × 109/l); hemoglobin, $92 \mathrm{~g} / \mathrm{l}$ (normal range $120-160 \mathrm{~g} / \mathrm{l}$ ), and platelets, $212 \times 10^{9} / \mathrm{l}$ (normal range 150-350 $\times 10^{9} / 1$ ). Unfortunately, further breathing difficulty developed in hospital, despite aggressive treatment with intravenous furosemide and albumin infusion. Therefore, the patient underwent bilateral RAE; pure alcohol mixed with lipidol was injected via the orifices of the right and left renal arteries to obliterate the arteries and their branches (fig. 1). After bilateral RAE, no proteinuria was noted, due to anuria. There was only mild nausea, flank pain, and mild fever for 1 day after embolization. The respiratory distress and anasarca edema subsided after regular HD. The serum albumin level was increased from 0.9 to $3.5 \mathrm{~g} / \mathrm{dl}$ within 3 months (fig. 2). An increase in muscle mass (the arm girth increased from 18 to $23 \mathrm{~cm}$, thigh girth from 25 to $35 \mathrm{~cm}$, waistline from 60 to $71 \mathrm{~cm}$, and dry weight from 46 to $57 \mathrm{~kg}$ ) was noted during the follow-up period. Hospitalization and protein substitution were no longer needed in the following 2 years (fig. 2). In addition, blood pressure was increased from about 70 80/50 60 to 100 120/60 70 mm Hg in the first year, and $120 \sim 135 / 70 \sim 80 \mathrm{~mm} \mathrm{Hg}$ in the second year after bilateral RAE.

\section{Discussion}

Although our case had developed severe proteinuria ( $>20 \mathrm{~g} /$ day), hypoalbuminemia $(1.4 \mathrm{~g} / \mathrm{dl})$, and anasarca edema under aggressive diuretic treatment, bed rest, fluid control, angiotensin converting enzymes, and nonsteroid anti-inflammatory drugs and albumin infusion, the patient and family agreed to bilateral RAE when the conditions worsened (urine protein $31.2 \mathrm{~g} /$ day and serum albumin $0.9 \mathrm{~g} / \mathrm{dl}$ ) and a pulmonary 
edema occurred. After bilateral RAE and following regular HD, the patient's refractory proteinuria, leg edema, and respiratory distress subsided (fig. 2). Because of the successful outcome in this case and previous reports [3-11], it is suggested that treatment with bilateral RAE as early as possible may be worthwhile for patients with severe proteinuria resistant to medical therapy.

In our case, concerns about the subsequent regular HD meant that the patient and family refused early bilateral RAE. The patient and family were anxious and wary of further loss of renal function and the stress of regular HD as a result of treatment with RAE. However, prolonged severe proteinuria resistant to medical therapy had caused the patient to reach a life-threatening cachexic state. In contrast, bilateral RAE and subsequent regular HD not only rapidly alleviated proteinuria, anasarca edema, and pulmonary edema, but also resulted in an increase in serum albumin level, with an obvious improvement in nutritional status, muscle mass and body weight, and clinical condition, with an obvious improvement in quality of life. The patient maintained a steady status for at least the next 2 years. In the meantime, no specific complications have occurred, except for the mild, self-limiting symptoms of post-infarction syndrome, which presented as mild flank pain, fever, and nausea for 1 day after the bilateral RAE. No specific complications arose as a result of the regular HD. This case and selected previous reports [3-11] reveal important information for physicians and patients in discussing prognoses and considering the pros and cons of bilateral RAE.

In conclusion, bilateral RAE is an alternative, effective, rapid, and safe procedure for the treatment of heavy proteinuria with nephrotic syndrome. Bilateral RAE with subsequent HD is useful in breaking a vicious circle and can improve nutritional status and alleviate the life-threatening complications associated with hypoalbuminemia.

\section{Disclosure Statement}

The authors have no conflicts of interest. 

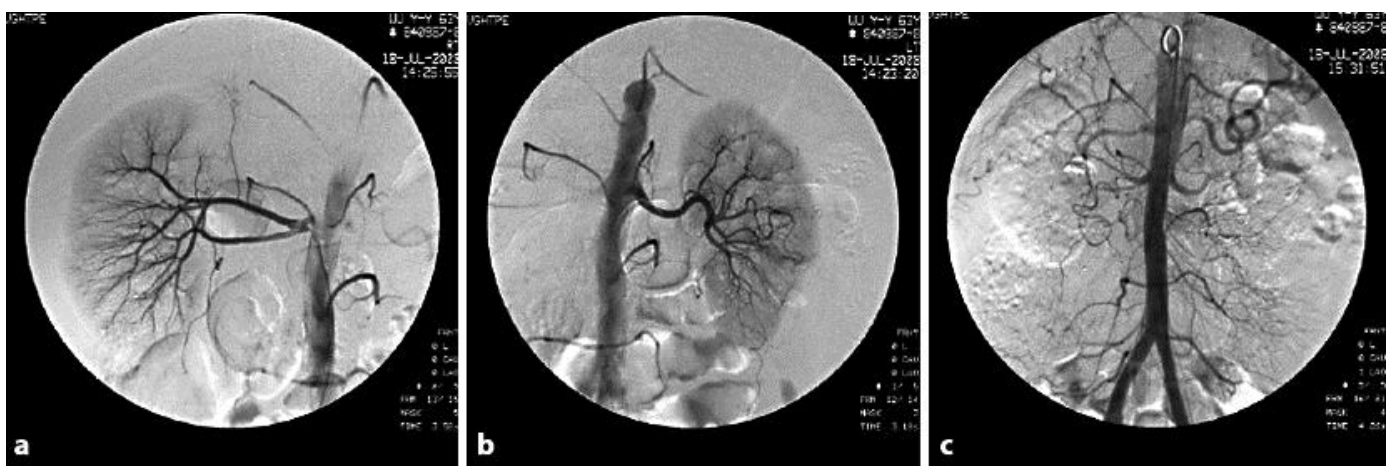

Fig. 1. Angiography and embolization of bilateral renal arteries. a, b Clear bilateral renal artery with its branches in angiography. c Obliteration of bilateral renal arteries and their branches after injection with pure alcohol mixed with lipidol via the orifice of the right and left renal artery.

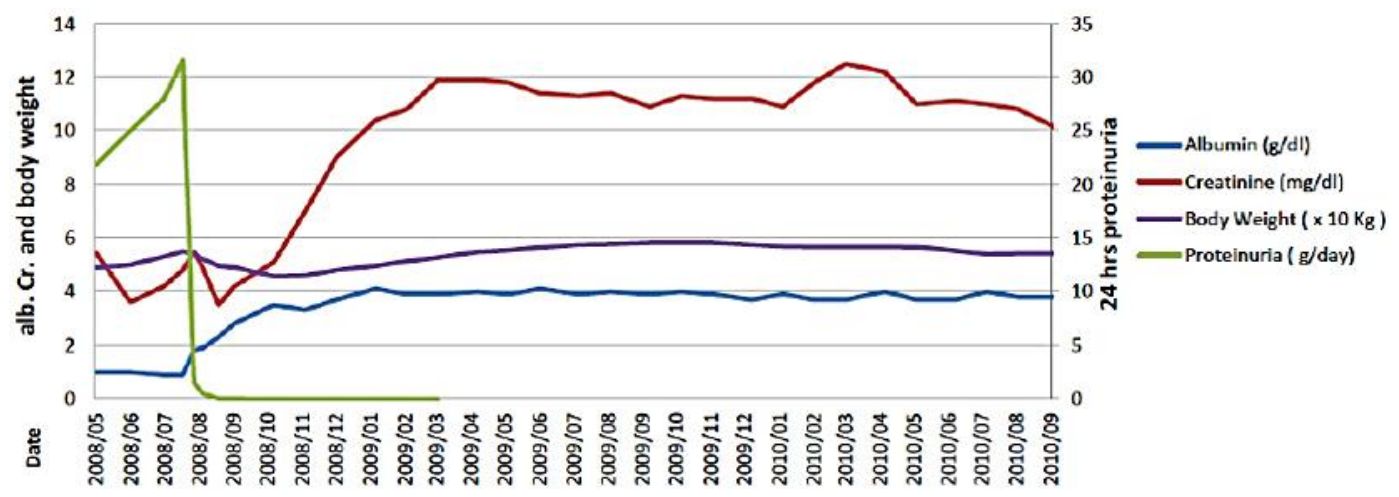

Fig. 2. The serial laboratory findings before and after bilateral RAE. Severe proteinuria relented after embolization on July 19, 2008. Serum albumin and creatinine levels and body weight (muscle mass) all increased after embolization and during HD over the 6 following months and remained at a relatively steady state thereafter.

\section{References}

-1 Schwartz MJ, Smith EB, Trost DW, et al: Renal artery embolization: clinical indications and experience from over 100 cases. BJU Int 2007;99:881-886.

-2 Capozza N, Collura G, Falappa P, et al: Renal embolization as an alternative to surgical nephrectomy in children. Transplant Proc 2007;39:1782-1784.

-3 Duboust A, Metivier F, Bedrossian J, Merland JJ: [Embolization of the renal arteries or 'medical nephrectomy' in amyloidosis nephrotic syndromes. 2 cases (author's transl)]. Nouv Presse Med 1979;8:3325-3327.

-4 Duda SH, Raible RT, Risler T, et al: [Therapeutic bilateral renal artery embolization in the nephrotic syndrome]. Dtsch Med Wochenschr 1994;119:58-62.

5 Solak Y, Polat I, Atalay H, et al: When urine is no longer beneficial: renal artery embolisation in severe nephrotic syndrome secondary to amyloidosis. Amyloid 2010;17:24-26.

-6 Turgut F, Kanbay M, Kaya A, et al: Bilateral renal artery embolization in a case with severe proteinuria secondary to amyloidosis in a hemodialysis patient. Amyloid 2007;14:157-158.

7 Kuhn C, Sonntag F, Runge M, et al: [Nephrotic syndrome as indication for one-stage bilateral renal embolisation (author's transl)]. Dtsch Med Wochenschr 1982;107:461-464. 
-8 Golwyn DH Jr, Routh WD, Chen MY, et al: Percutaneous transcatheter renal ablation with absolute ethanol for uncontrolled hypertension or nephrotic syndrome: results in 11 patients with end-stage renal disease. J Vasc Interv Radiol 1997;8:527-533.

99 Olivero JJ, Frommer JP, Gonzalez JM: Medical nephrectomy: the last resort for intractable complications of the nephrotic syndrome. Am J Kidney Dis 1993;21:260-263.

10 Prokopiuk-Wierzbicka M, Wierzbicki P, Perkowska-Ptasinska A, et al: [Severe nephrotic syndrome requiring bilateral renal arteries embolisation in patient with membranous and diabetic nephropathy]. Pol Merkur Lekarski 2008;24:321-324.

11 Tikkakoski T, Leppanen M, Turunen J, et al: Percutaneous transcatheter renal embolization with absolute ethanol for uncontrolled nephrotic syndrome. Case reports. Acta Radiol 2001;42:80-83.

12 Dingwall RR: Living with renal failure: the psychological issues. EDTNA ERCA J 1997;23:28-30, 35.

13 Goldwasser P, Feldman J: Association of serum albumin and mortality risk. J Clin Epidemiol 1997;50:693-703.

14 Schalk BW, Visser M, Bremmer MA, et al: Change of serum albumin and risk of cardiovascular disease and all-cause mortality: Longitudinal Aging Study Amsterdam. Am J Epidemiol 2006;164:969-977. 